\title{
Corrección del componente rotacional prequirúrgico y postquirúrgico del primer metatarsiano secundario a osteotomía de Chevron modificado
}

\author{
Correction of the pre- and post-surgical rotational component of the \\ first metatarsal secondary to modified Chevron osteotomy \\ Espinosa-Uribe AG, ${ }^{*, \ddagger}$ Ortiz-Garza JI, ${ }^{\ddagger, \S}$ García-Limón M, ${ }^{\ddagger, \S}$ \\ Fernández-Garza F, ${ }^{\S}$ Valverde-Galindo LA, ${ }^{*, \ddagger}$ Meza Flores J, ${ }^{*, \ddagger}$ Gutiérrez-de la OJף \\ Departamento de Ortopedia y Traumatología, Hospital Christus Muguerza.
}

RESUMEN. Introducción: Hallux valgus (HV) es una patología compleja del antepié, recientemente se ha mostrado interés en el componente rotacional (CR) distal del primer metatarsiano por su relación con recidivas postquirúrgicas de la deformidad. La selección de la técnica quirúrgica es controversial con escasa literatura acerca de la corrección de rotación con osteotomías comunes. Nuestro objetivo es evaluar características radiológicas prequirúrgicas y postquirúrgicas respecto a CR del primer metatarsiano en la cirugía de corrección de HV moderado a severo usando osteotomía de Chevron largo. Material y métodos: Estudio observacional, prospectivo y comparativo en el que se evaluó el CR del primer metatarsiano en nueve pies pertenecientes a seis pacientes con una edad media de 45 años. El CR fue evaluado con base en la metáfisis proximal del primer metatarsiano en fluoroscopías axiales sin carga y AP de pie. Las mediciones se realizaron por un residente de ortopedia y traumatología y un subespecialista en cirugía de pie y Tobillo. Resultados: Se documentaron diferencias significativas $(\mathrm{p}=0.05)$ en el ángulo de pronación prequirúrgico y postquirúrgico al utilizar osteotomía de Chevron largo. El resultado funcional de los pacientes evaluados fue excelente a 11 meses de seguimiento. Con-
ABSTRACT. Introduction. Hallux valgus (HV) is a complex forefoot pathology, in which interest has recently been shown in the distal rotational component (CR) of the first metatarsal due to its relationship with postsurgical relapses of the deformity. The selection of surgical technique is controversial with little current literature on the correction of rotation with common osteotomies. The aim of this study is to evaluate preoperative and postoperative radiological characteristics regarding CR of the first metatarsal using long Chevron osteotomy for moderate to severe HV. Material and methods: An observational, prospective and comparative study was carried out in which the CR of the first metatarsal was evaluated in nine feet belonging to six patients with a mean age of 45 years. The RC was evaluated based on the proximal metaphysis of the first metatarsal in axial fluoroscopies of unloaded metatarsals and standing AP. Measurements were performed by an Orthopedic and Traumatology resident and a Foot and Ankle Surgery subspecialist. Results: Significant differences $(p=0.05)$ were documented in the preoperative and postoperative pronation angle when using long Chevron osteotomy. The functional result of the evaluated patients was excellent

\section{Nivel de evidencia: IV}

* Universidad de Monterrey, Escuela de Medicina, Especialidades Médicas.

* Departamento de Ortopedia y Traumatología, Hospital Christus Muguerza, Alta Especialidad.

${ }^{\S}$ Instituto de Seguridad Social de Trabajadores del Estado de Nuevo León (ISSSTELEON). Monterrey, Nuevo León, México.

" Departamento de Anatomía Humana, Facultad de Medicina y Hospital Universitario «Dr. José E González», Universidad Autónoma de Nuevo León.

Correspondencia:

Jaime Isaías Ortiz-Garza

Hospital Christus Muguerza Alta Especialidad

Ave. Hidalgo Núm. 2525, Col. Obispado, C.P. 64460, Monterrey, Nuevo León, México.

E-mail: drjaimeortiz@outlook.com

Citar como: Espinosa-Uribe AG, Ortiz-Garza JI, García-Limón M, Fernández-Garza F, Valverde-Galindo LA, Meza FJ, et al. Corrección del componente rotacional prequirúrgico y postquirúrgico del primer metatarsiano secundario a osteotomía de Chevron modificado. Acta Ortop Mex. 2020; 34(6): 354-358. https://dx.doi.org/10.35366/99131

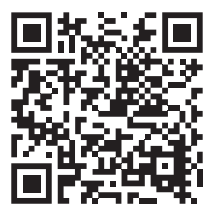


clusiones: Radiológicamente, la corrección rotacional es variable y previamente no se ha reportado en la literatura con esta osteotomía. Se establecen bases morfológicas para la realización de estudios posteriores en la evaluación de rotación de cabeza del primer metatarsiano con diferentes osteotomías.

Palabras clave: Componente rotacional, hallux valgus, pronación de metatarsiano, osteotomía de Chevron, osteotomía correctiva.

\section{Introducción}

La deformidad del hallux valgus, coloquialmente conocida como «juanete», constituye una patología que comúnmente afecta a mujeres de mediana edad, con consecuencias estéticas, sicológicas y funcionales en los pacientes. ${ }^{1,2}$ Dicha deformidad del primer metatarsiano constituye una patología progresiva en tres planos del antepié, caracterizada por una desviación lateral del hallux y con desviación medial y pronación de la cabeza correspondiente al primer metatarsiano. ${ }^{3,4,5}$ Se ha descrito como una patología subdiagnosticada debido a que corresponde a un espectro de manifestaciones clínicas desde la deformidad simple hasta deformidades severas cursando con dolor y limitación funcional del antepié y de la extremidad inferior.

En el manejo quirúrgico del hallux valgus se han descrito más de 200 diversas técnicas quirúrgicas desde liberación percutánea de tejidos blandos hasta osteotomías percutáneas o abiertas proximales o distales en el metatarsiano con diferencias en el desenlace radiológico y clínico del paciente. ${ }^{6,7,9}$ Dichas técnicas se han desarrollado principalmente con la intención de corregir la deformidad en el plano frontal; sin embargo, recientemente se ha mencionado la importancia del componente rotacional en el desenlace clínico y radiológico del paciente. ${ }^{2,10}$

En tiempos recientes se han descrito osteotomías rotacionales del primer metatarsiano con alto poder de corrección de la pronación en la deformidad del hallux; ;,3,10,11 sin embargo, algunas constituyen técnicas demandantes cuando el cirujano at 11 months of follow-up. Conclusions: Radiologically, the rotational correction is variable and has not previously been reported in the literature with this osteotomy. We establishes the morphological bases for the performance of subsequent studies in the evaluation of head rotation of the 1 st metatarsal with different osteotomies.

Keywords: Chevron osteotomy, correction osteotomy, hallux valgus, metatarsus pronation, morphology.

no está familiarizado con ellas. Asimismo, existe un área de controversia en el poder de corrección de osteotomías convencionales comúnmente conocidas en el manejo del hallux valgus como la osteotomía de Chevron, ${ }^{8,12}$ las cuales disminuyen el tiempo quirúrgico y brindan mayor comodidad al cirujano, el cual ya está familiarizado con la técnica.

El objetivo del presente estudio es documentar cambios prequirúrgicos y postquirúrgicos en la rotación de la cabeza del primer metatarsiano secundarios a la osteotomía de Chevron modificada.

\section{Material y métodos}

Se realizó un estudio observacional, longitudinal y comparativo en el que se documentaron características radiológicas del componente rotacional (CR) del primer metatarsiano en nueve pies pertenecientes a seis pacientes en radiografías axiales de metatarsianos relacionadas con la rotación de la cabeza del metatarsiano en radiografías anteroposteriores de pie asociándolos con pronación de la cabeza del primer metatarsiano en radiografías axiales de metatarsianos. Se incluyeron pacientes de nacionalidad mexicana de cualquier edad y género, los cuales fueron sometidos a cirugía primaria de hallux valgus sin importar antecedente de otras patologías y/o comorbilidades de antepié. En dichos pacientes la deformidad fue clasificada de moderada a severa con un IMA (ángulo intermetatarsal) mayor o igual a 12 PASA (proximal articular set angle) mayor o igual a ocho) sin importar fórmula metatarsal.
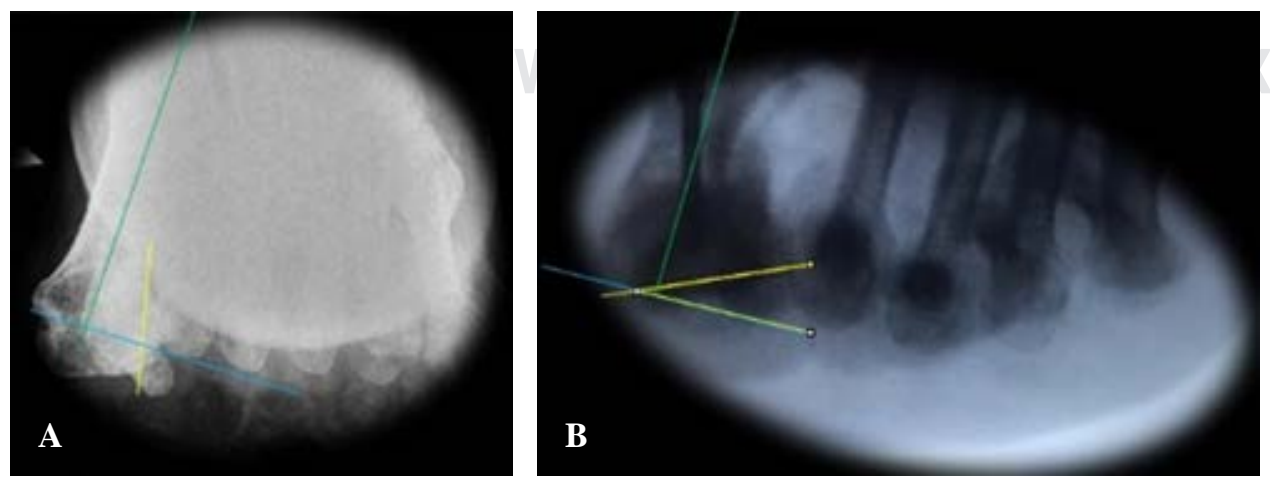

Figura 1:

Evaluación del componente rotacional pre- y postquirúrgico aplicando el método propuesto por Mortier JP et al., utilizando la herramienta goniómetro de ImageJ. https://imagej.nih.gov/. 


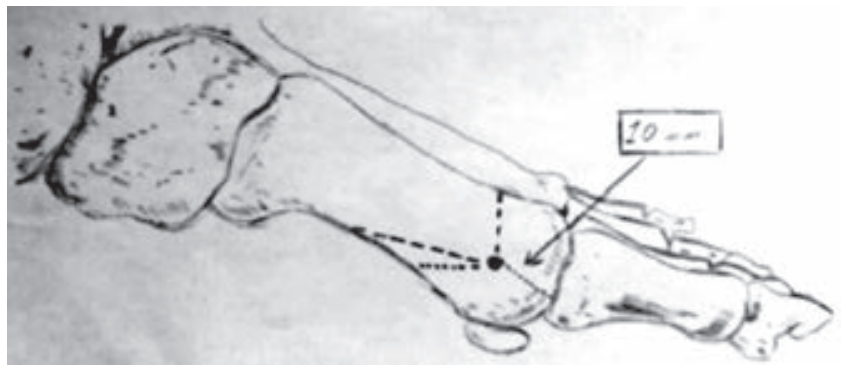

Figura 2: Imagen representativa de la osteotomía de Chevrón con brazo plantar corto y largo.

Se excluyeron pacientes con cirugías previas de hallux valgus hipermovilidad del primer metatarsiano y pacientes que no desearan ser incluidos en el estudio. Durante el estudio se eliminaron tres pacientes, los cuales contaban con estudios incompletos o radiografías tomadas con mala técnica.

\section{Procedimiento}

Se incluyeron mediciones radiográficas previas y posteriores al procedimiento con los ángulos correspondientes al hallux valgus: IMA, ángulo del hallux valgus PASA, distal articular set angle (DASA) y CR del primer metatarsiano. Se utilizaron recursos matemáticos y estadísticos para analizar los datos y determinar una correlación entre los datos obtenidos por dos observadores utilizando estadística descriptiva y gráfica.

Las proyecciones anteroposteriores se realizaron con el pie apoyado sobre el tambor del fluoroscopio. Para las proyecciones axiales se colocará el rayo paralelo a los metatarsianos. El CR de la cabeza del primer metatarsiano fue evaluado utilizando el método descrito por Mortier y colaboradores en el que se evalúa la rotación de la cabeza respecto a una línea de la diáfisis del primer metatarsiano. ${ }^{13}$ Esta técnica de medición en proyecciones axiales de sesamoideos es relevante, ya que considera la diáfisis del primer metatarsiano respecto a otra línea que intercepta las corticales plantares de los surcos sesamoideos medial y lateral (Figura 1). Nuestro equipo de trabajo previamente ha determinado correlaciones intraclase estadísticamente significativas $(\mathrm{p}=0.02)$ en la evaluación del componente rotacional por tres observadores que utilizan esta técnica de medición en 81 pies de 48 pacientes usando sólo radiografías prequirúrgicas (resultados no publicados).

\section{Descripción de la técnica quirúrgica}

Se realizó la técnica quirúrgica de osteotomía de Chevron largo descrita en estudios previos en nuestro servicio por Reyes-Terán y colaboradores. Se abordó longitudinalmente sobre la superficie medial, abarcando desde la proximidad de la primera falange hasta observar la diáfisis del primer metatarsiano. Se realizó el corte capsular longitudinal, conservando el plano para su posterior plicatura y reparación. Se continuó con la buniectomía.
La osteotomía de Chevron modificada consiste en un corte en «V» del primer metatarsiano, con una arista larga plantar y una corta dorsal. El ápice debe situarse a $10 \mathrm{~mm}$ de la superficie articular (Figura 2). La cabeza del metatarsiano se desplaza lateralmente y se fija con un tornillo canulado $2.0 \mathrm{~mm}$ de dorso-medial a plantar-lateral. Posteriormente se realiza la osteotomía de Akin fijándose de manera externa con un vendaje correctivo.

Posteriormente se documentaron y evaluaron las mismas mediciones de ángulo de hallux valgus, IMA, MTF y PASA, así como el CR en fluoroscopías de control postquirúrgicas utilizando la misma técnica que en la evaluación preoperatoria.

Se obtuvieron media y desviación estándar para cada parámetro de medición por género y grupo de edad para realizar la estadística descriptiva. Además, se hicieron pruebas de Shapiro-Wilk para determinar la distribución de los datos así como t de Student, muestras pareadas y prueba de Wilcoxon no paramétrica al evaluar las variables morfométricas de ángulo intermetatarsiano, ángulo del hallux, proximal articular set angle (PASA) y para la diferencia de rotación de la cabeza del primer metatarsiano prequirúrgica y postquirúrgica. Se correlacionó el ángulo de la cabeza del primer metatarsiano respecto al PASA utilizando un coeficiente de correlación intraclase. En el análisis univariado, las variables cuantitativas se reportaron en media y desviación estándar o mediana y rango intercuartil de acuerdo a si su distribución fue paramétrica o lo contrario, variables cualitativas fueron reportadas en frecuencia y porcentaje. Se estimó el tamaño del efecto calculando la D de Cohen (D = Media 1-Media 2/desviación estándar ponderada) en las comparaciones paramétricas. En las comparaciones no paramétricas el tamaño del efecto se interpretó con el valor de $R^{2}(r=Z / N)$. Para estimar una posible correlación entre el cambio en el ángulo de pronación y el cambio en las angulaciones medidas adicionalmente (ángulo intermetatarsiano DASA, PASA, etcétera) se utilizó la prueba de correlación de Spearman para muestras no paramétricas.

Un valor de p menor de 0.05 se consideró estadísticamente significativo para cada uno de los parámetros de medición. Se utilizó el programa computacional SPSS versión 24.0 para Windows (IBM, Armonk, NY, USA).

No fue necesaria la identificación del paciente con datos ni ninguna información personal para el propósito del estudio (excepto género y edad). Esto para asegurar el cumplimiento de la NOM-004-SSA3-2012. Debido a que el presente protocolo corresponde a un estudio en el que los datos se evaluarán de manera retrospectiva, no intervendrá de ninguna forma con el manejo hospitalario o en consulta externa de los pacientes. El presente estudio fue previamente aprobado por el comité de ética de nuestro hospital con el número de aprobación 23052019-CM-CI.

\section{Resultados}

Se evaluaron nueve pies pertenecientes a seis pacientes, la edad media de los pacientes evaluados fue de $45.75 \pm 19.24$, 
Tabla 1: Comparación de angulaciones prequirúrgicas y postquirúrgicas.

$\begin{array}{llccc}\text { Variable } & & \text { Angulación } & \text { Tamaño del efecto } & 0.35 \\ \text { Ángulo intermetatarsiano } & \text { Prequirúrgico } & 16.81 \pm 9.01 & 0.380 & 1.13 \\ \text { Ángulo hallux } & \text { Postquirúrgico } & 10.21 \pm 1.64 & 0.050 & 1.7 \\ & \text { Prequirúrgico } & 24.52 \pm 10.19 & 14.3 \pm 6.87 & 0.024 \\ \text { Ángulo DASA } & \text { Postquirúrgico } & 5.86 \pm 2.15 & 0.077 & 0.99 \\ \text { Ángulo PASA } & \text { Prequirúrgico } & 3.48 \pm 1.36 & 0.050 & -0.25\left(\mathrm{r}^{2}=0.06\right)\end{array}$

Resultados reportados en media y desviación estándar (distribución paramétrica) [prueba t de Student para muestras relacionadas]. *Reportados en mediana y rango intercuartil (distribución no paramétrica) [prueba de Wilcoxon].

PASA = proximal articular set angle, DASA $=$ distal articular set angle, $\mathrm{CR}=$ componente rotacional.

$100 \%$ de la muestra corresponde a género femenino, la media de tiempo de evolución fue de $4.16 \pm 2.87$ previo a la cirugía.

Las características descriptivas de las angulaciones prequirúrgicas y postquirúrgicas de la muestra evaluada se enumeran en la Tabla 1.

La correlación de cambio de las variables respecto a la intervención con osteotomía de Chevron modificada se describe en la Tabla 2. La variabilidad interobservador se muestra en la Tabla 3.

Adicionalmente se documentó un seguimiento promedio de 11 meses para los pacientes evaluados. El tiempo de consolidación promedio fue de 3.2 meses. De la presente serie se documentó pérdida de la reducción y fijación en un caso (un pie) secundario a convulsiones idiopáticas de la paciente durante las primeras horas del período postoperatorio inmediato así como otro caso aislado de intolerancia de material de osteosíntesis (un pie). La totalidad de la muestra evaluada se reintegró a actividades laborales seis meses posteriores a las intervenciones quirúrgicas.

\section{Discusión}

Se documentaron diferencias estadísticamente significativas en el CR prequirúrgico y postquirúrgico al utilizar osteotomía de Chevron largo con disminución en dicho componente (Tabla 1). Dichos resultados son relevantes técnica y clínicamente, ya que para nuestro conocimiento el poder de corrección rotacional de la osteotomía de Chevron con brazo plantar largo no ha sido reportado previamente en la literatura.

La relevancia técnica radica en la rotación y sobreposición de las corticales al momento de realizar la reducción y fijación de la osteotomía. Sin embargo, dicha corrección hasta el momento es variable dependiendo de la maniobra y rotación empleada por el cirujano al corregir la deformidad.

Desde su introducción hace más de 40 años la osteotomía de Chevron ha ganado aceptación en los cirujanos de pie y tobillo como uno de los procedimientos que con más frecuencia se realizan mundialmente para el tratamiento de hallux valgus. ${ }^{11}$ Dicha técnica original ha recibido modificaciones, las cuales han permitido mayor nivel de corrección principalmente en el IMA y DMAA. ${ }^{14}$ Recientemente Prado y colaboradores describieron la modificación de la osteotomía de Chevron con cuñas de sustracción en la cortical medial, las cuales permiten hacer correcciones en el CR del primer metatarsiano. ${ }^{11}$ Sin embargo, en su descripción técnica no menciona el poder de corrección del CR de dicha osteotomía. En nuestra experiencia al realizar osteotomía de Chevron largo sin el uso de cuñas de sustracción hemos documentado valores de corrección variables.

Es bien conocido en la literatura que el tipo de osteotomía influye en el grado de corrección respecto a (osteotomías rotacionales) previamente descritas en notas técnicas. ${ }^{10,11}$ Sin embargo, realizar osteotomías previamente descritas con la intención de rotar la cabeza del metatarsiano podría disminuir las posibles complicaciones relacionadas con las modificaciones propuestas ${ }^{3,15}$ así como disminuir tiempo quirúrgico, ya que el cirujano se encuentra ya familiarizado con una técnica bien aceptada en la práctica clínica como la osteotomía de Chevron, aunque con variantes modificadas.

El presente estudio describe la reducción del CR del primer metatarsiano al utilizar osteotomía de Chevron largo. La principal limitación de este estudio es la cantidad de pacientes reclutados así como la precisión y poder del efecto de la muestra evaluada. Asimismo, una de las principales limitaciones y controversias del estudio que nos ocupa es el uso de proyecciones sin carga para la evaluación del CR. Es importante mencionar que no existe un consenso claro en estudios previos $2,5,14,16,17$ acerca de la cantidad de carga que debe utilizarse para evaluar el CR.

Sin embargo, este estudio aporta las bases para la realización de estudios subsecuentes en los cuales sea posible evaluar cambios prequirúrgicos y postquirúrgicos al utilizar diferentes técnicas de osteotomías para la corrección de HV y su evaluación en proyecciones axiales de metatarsianos así como en estudios morfológicos que evalúen la pronación de la cabeza del primer metatarsiano. Dicha información sería de utilidad en la comprensión de la fisiopatología del hallux valgus. 
Tabla 2: Correlación de cambio en ángulo de pronación con los otros cambios en angulación utilizando prueba de U de Mann-Whitney.

$\begin{array}{lcc}\text { Ángulo } & \begin{array}{c}\text { Coeficiente } \\ \text { de correlación }\end{array} & \mathrm{p} \\ \text { Intermetatarsiano } & 0.357 & 0.380 \\ \text { Hallux } & -0.143 & 0.736 \\ \text { Distal articular set angle } & 0.333 & 0.420 \\ \text { Proximal articular set angle } & 0.429 & 0.289 \\ \text { Se utilizó la prueba de correlación de Spearman para variables cuantitativas } \\ \text { no paramétricas (se usó esa porque la variable del ángulo de pronación es no } \\ \text { paramétrica). }\end{array}$

\begin{tabular}{|c|c|c|}
\hline \multicolumn{3}{|c|}{$\begin{array}{l}\text { Tabla 3: Coeficiente de correlación intraclase para } \\
\text { mediciones realizadas por dos observadores. }\end{array}$} \\
\hline Variable & & Coeficiente \\
\hline \multirow[t]{2}{*}{ Ángulo intermetatarsiano } & Prequirúrgico & 0.287 \\
\hline & Postquirúrgico & 0.033 \\
\hline \multirow[t]{2}{*}{ Ángulo hallux } & Prequirúrgico & 0.130 \\
\hline & Postquirúrgico & 0.631 \\
\hline \multirow[t]{2}{*}{ Ángulo DASA } & Prequirúrgico & 0.834 \\
\hline & Postquirúrgico & 0.300 \\
\hline \multirow[t]{2}{*}{ Ángulo PASA } & Prequirúrgico & 0.870 \\
\hline & Postquirúrgico & 0.860 \\
\hline \multirow[t]{2}{*}{ Ángulo de pronación } & Prequirúrgico & 0.990 \\
\hline & Postquirúrgico & 0.980 \\
\hline
\end{tabular}

\section{Conclusiones}

Se documentaron diferencias significativas $(\mathrm{p}<0.05)$ en el ángulo de pronación prequirúrgico y postquirúrgico al utilizar osteotomía de Chevron. Radiológicamente dicha corrección rotacional es variable y no se ha reportado con anterioridad en la literatura. Los pacientes tuvieron consolidación y excelente desenlace funcional a un año de seguimiento.

El presente estudio establece bases morfológicas para la realización de estudios posteriores en la evaluación de rotación de cabeza del primer metatarsiano con diferentes osteotomías.

\section{Agradecimientos}

Agradecemos a la Dra. C. María Elena Romero por su apoyo en el análisis estadístico. Adicionalmente agradece- mos el apoyo de Dr. Jorge Luis Reyes-Terán por sus importantes comentarios técnicos durante la realización de los procedimientos.

\section{Referencias}

1. Wagner P, Wagner E. Is the rotational deformity important in our decision- making process for correction of hallux valgus deformity? Foot Ankle Clin. 2018; 23(2): 205-17.

2. Saltzman CL, Brandser EA, Anderson CM, Berbaum KS, Brown TD. Coronal plane rotation of the first metatarsal. Foot Ankle Int. 1996; 17(3): 157-61.

3. Wagner E, Wagner P. Metatarsal pronation in hallux valgus deformity: a review. J Am Acad Orthop Surg Glob Res Rev. 2020; 4(6): e20.00091. doi: 10.5435/jaaosglobal-d-20-00091.

4. Hatch DJ, Santrock RD, Smith B, Dayton P, Weil L. Triplane hallux abducto valgus classification. J Foot Ankle Surg. 2018; 57(5): 972-81.

5. Smith WB, Santrock RD, Hatch DJ, Dayton P. Intraoperative mulitplanar alignment system to guide triplanar correction of hallux valgus deformity. Tech Foot Ankle Surg. 2017; 16: 175-82.

6. Easley ME, Trnka H. Current concepts review: hallux valgus part 1: pathomechanics, clinical assessment, and nonoperative management mark. Foot Ankle Int. 2007; 28(5): 654-9.

7. Easley ME, Trnka H. Current concepts review: hallux valgus part II : operative treatment. Foot Ankle Int. 2007; 28: 748-57.

8. Lee KB, Cho NY, Seon JK. A comparison of proximal and distal Chevron osteotomy, both with lateral soft-tissue release, for moderate to severe hallux valgus in patients undergoing simultaneous bilateral correction. Bone Joint J. 2015; 97: 202-7.

9. Sharma J, Aydogan U. Algorithm for severe hallux valgus associated with metatarsus adductus. Foot Ankle Int 2015; 36: 1499-503.

10. Wagner P, Ortiz C, Wagner E. Rotational osteotomy for hallux valgus. A new technique for primary and revision cases. Tech Foot Ankle Surg. 2017; 16(1): 3-10.

11. Prado M, Baumfeld T, Nery C, Mendes A, Baumfeld D. Rotational biplanar Chevron osteotomy. Foot Ankle Surg. 2020; 26(4): 473-6. doi: 10.1016/j.fas.2019.05.011.

12. Jung HG, Kim TH, Park JT, Shin MH, Lee SH. Proximal reverse Chevron metatarsal osteotomy, lateral soft tissue release, and akin osteotomy through a single medial incision for hallux valgus. Foot Ankle Int. 2014; 35: 368-73.

13. Mortier JP, Bernard JL, Maestro M. Axial rotation of the first metatarsal head in a normal population and hallux valgus patients. Orthop Traumatol Surg Res. 2012; 98(6): 677-83.

14. Nery C, Barroco R, Réssio C. Biplanar Chevron osteotomy. Foot Ankle Int. 2002; 23(9): 792-8.

15 Midjord J. Bunion surgery: check these radiographic parameters intraoperatively to avoid recurrence: Commentary on an article by Chul Hyun Park, MD, PhD, and Woo-Chun Lee, MD, PhD: "recurrence of hallux valgus can be predicted from immediate postoperative non-weight-bearing radiographs". J Bone Joint Surg Am. 2017; 99(14): e80.

16. Cruz EP, Wagner FV, Henning C, Veiga SJ, Pagnussato F, Galia CR Comparison between simple radiographic and computed tomographic three-dimensional reconstruction for evaluation of the distal metatarsal articular angle. J Foot Ankle Surg. 2017; 56(3): 505-9.

17. Dayton P, Feilmeier M, Hirschi J, Kauwe M, Kauwe JSK. Observed changes in radiographic measurements of the first ray after frontal plane rotation of the first metatarsal in a cadaveric foot model. $J$ Foot Ankle Surg. 2014; 53: 274-8.

Conflicto de intereses: Los autores declaran no tener ningún conflicto de interés por la realización del presente estudio. 